\section{(6) OPEN ACCESS}

\title{
Evaluation of WHO screening algorithm for the presumptive treatment of asymptomatic rectal gonorrhoea and chlamydia infections in at-risk MSM in Kenya
}

\author{
Eduard J Sanders, ${ }^{1,2}$ Elizabeth Wahome, ${ }^{1}$ Haile Selassie Okuku, ${ }^{1}$ Alexander N Thiong ${ }^{\prime} 0,{ }^{1}$ \\ Adrian D Smith, ${ }^{3}$ Sarah Duncan, ${ }^{4}$ John Mwambi, ${ }^{1}$ Juma Shafi, ${ }^{5,6}$ R Scott McClelland, ${ }^{5,6}$ \\ Susan M Graham ${ }^{1,5,6}$
}

${ }^{1}$ Centre for Geographic Medicine Research - Coast, Kenya Medical Research Institute (KEMRI), Kilifi, Kenya ${ }^{2}$ Nuffield Department of Clinical Medicine, University of Oxford, Headington, UK ${ }^{3}$ Department of Public Health, University of Oxford, Headington, UK

${ }^{4}$ The Churchill Hospital, University Hospitals, Oxford, UK

${ }^{5}$ University of Nairobi, Nairobi, Kenya

${ }^{6}$ University of Washington, Seattle, Washington, USA

Correspondence to Dr Eduard I Sanders, Kenya Medical Research Institute, Centre for Geographic Medicine Research-Coast, PO Box 230, Kilifi, Kenya; ESanders@kemri-wellcome.org

Received 2 February 2013 Revised 19 September 2013 Accepted 9 October 2013 Published Online First 10 December 2013

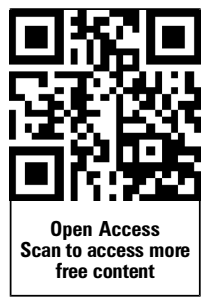

To cite: Sanders $E J$, Wahome E, Okuku HS, et al. Sex Transm Infect 2014;90:94-99.

\begin{abstract}
Objectives The WHO recommends that men who have sex with men (MSM) reporting unprotected receptive anal intercourse (RAI) and either multiple partners or a partner with a sexually transmitted infection (STI) in the past 6 months should be presumptively treated for asymptomatic rectal Neisseria gonorrhoeae (NG) and Chlamydia trachomatis (CT) infections. We evaluated this recommendation in a cohort of 'high-risk' MSM in Coastal Kenya.

Methods We assessed presence of genitourinary and rectal symptoms, and determined prevalence and 3-month incidence of rectal NG and CT infections. We performed nucleic acid amplification testing of urine and rectal swab samples collected from MSM followed

either multiple sex partners or a sex partner with a sexually transmitted infection (STI) in the past 6 months should be presumptively treated for rectal Neisseria gonorrhoeae (NG) and Chlamydia trachomatis (CT) infections. ${ }^{2}$ This WHO recommendation has not been evaluated in resourcelimited countries, nor has it been mentioned in WHO's renewed commitment to STI prevention and control in achieving global sexual and reproductive health. ${ }^{3}$ A recent study among MSM sex workers in Cote D'Ivoire, finding a high burden of rectal NG, called for validation of the WHO algorithm. ${ }^{4}$ We evaluated this recommendation in a cohort of MSM followed for HIV-1 and STI acquisition risks in Coastal Kenya. ${ }^{5} 6$
\end{abstract} prospectively, and assessed predictive values of the WHO algorithm at baseline screening.

Results Of 244 MSM screened, 240 (98.4\%) were asymptomatic, and $147(61.3 \%)$ reported any RAI in the past 6 months. Among 85 (35.4\%) asymptomatic MSM meeting criteria for the WHO presumptive treatment (PT) recommendation, we identified 20 with rectal infections (six NG, 12 CT and two NG-CT co-infections). Among 62 asymptomatic MSM who did not meet criteria, we identified seven who were infected. The sensitivity and specificity of the WHO algorithm were $74.1 \%(95 \% \mathrm{Cl}$ $53.7 \%$ to $88.9 \%)$ and $45.8 \%(95 \% \mathrm{Cl} 36.7 \%$ to $55.2 \%)$, respectively. The 3 -month incidence of any rectal NG or CT infection in asymptomatic men reporting any RAI was 39.7 (95\% Cl 24.3 to 64.8) per 100 person-years.

Conclusions About one-third of asymptomatic MSM were eligible to receive PT for NG and CT infections. Among MSM who would qualify for PT of rectal STIS, the number needed to treat in order to treat one infection was four. Our results support the value of the WHO screening algorithm and recommended PT strategy in this population.

\section{INTRODUCTION}

Men who have sex with men (MSM) in Africa require urgent interventions to reduce acquisition and transmission of HIV-1, but focused approaches are yet to be implemented. ${ }^{1}$ In 2011, the WHO recommended that asymptomatic MSM reporting unprotected receptive anal intercourse (RAI) and

\section{METHODS}

Between July and October 2011, HIV-1 negative and positive MSM in follow-up in previously described cohort studies in Coastal Kenya ${ }^{5}{ }^{6}$ were screened for NG and CT using a nucleic acid amplification test (NAAT; Gen-Probe Aptima Combo 2 assay, San Diego, California, USA). ${ }^{7}$ MSM were recruited for these cohort studies if they reported multiple sex partners, anal intercourse, sex work or an STI in the past 3 months. MSM who were HIV-1 negative at screening were enrolled in an open HIV-1 vaccine feasibility cohort ${ }^{5}$; MSM who were HIV-1 positive at screening were enrolled in a parallel cohort to receive HIV-1 care at the same research clinic. ${ }^{8}$ Enrolled men received quarterly risk-reduction counselling and HIV-1 testing (if previously seronegative), and had a medical history and physical examination at each scheduled visit. Face-to-face interviews were used to ascertain sexual risk behaviour and determine if WHO criteria for presumptive treatment (PT) were met. As cohort subjects had quarterly risk assessments, we used routinely collected cohort data to establish whether RAI was unprotected. For each subject, we determined whether condoms were used with the last sexual partner, with up to three identifiable partners in the previous month, and for all RAI over the previous 3-month period. For MSM who were in follow-up for 6 months or more $(>75 \%$ of total), we also included data from the preceding quarterly cohort visit to establish whether RAI was 
unprotected over the 6-month period targeted by the WHO guidelines. At each cohort visit, men were asked if they had dysuria, urethral or rectal discharge, or rectal pain. All men submitted a urine specimen and had a rectal swab collected by a clinician. ${ }^{9}$ Each sample was tested for NG and CT using the Aptima Combo 2 assay. Men with rectal or urethral symptoms compatible with infection or NAAT-confirmed infections received cefixime (400 $\mathrm{mg}$ immediately) and doxycycline (100 mg twice a day for 7 days), risk-reduction counselling, and advice on partner treatment. Patients were given the option to take medication for their sex partner(s) or refer their partner(s) to the research clinic for treatment. Men reporting any RAI were invited for rescreening for urethral and rectal NG and CT infections at their scheduled quarterly visit.

\section{Data analysis}

Sociodemographic and behavioural risk factors for asymptomatic (prevalent) rectal NG or CT infections were summarised for men reporting any RAI. Categorical variables were tested using $\chi^{2}$ tests. Prevalence ratios were used to measure associations between potential risk factors and baseline NG or CT infection. A multivariable exact poisson regression model was used to estimate adjusted prevalence ratios. We calculated the area under the receiver operator characteristic curve (AUC) for the predictive ability of the WHO algorithm and alternative risk criteria to identify patients with asymptomatic rectal infections for PT. The 3-month incidence rate of any rectal NG or CT acquisition was expressed as incidence per 100 person-years (PY). Cox proportional hazards models were used to assess risk factors for incident rectal NG or CT infection.

\section{RESULTS}

A total of 244 MSM had a urine and rectal sample collected for evaluation at baseline, of whom four (1.6\%) had a symptomatic STI, including one with urethral discharge, one with dysuria, one with rectal pain, and one with rectal pain and discharge. Of the four symptomatic infections, three (75\%) were NAAT-confirmed (two NG and one NG-CT co-infection, table 1). Of 240 asymptomatic men, 147 (61.2\%) reported any RAI in the past 6 months, and $93(38.8 \%)$ did not report any RAI. Overall, $28(11.7 \%)$ of 240 asymptomatic men had an anogenital infection diagnosed. In 147 MSM reporting any RAI, 27 (18.4\%) had anogenital infections, including all rectal NG infections and all but one rectal CT infections. In 93 MSM not reporting any RAI, one $(1.1 \%$ ) had a rectal CT infection (table $1)$. Upon chart review of the latter patient, RAI had been

Table 1 Evaluation of the WHO screening algorithm for presumptive treatment of asymptomatic rectal gonorrhoea and chlamydia infections in 244 MSM, Coastal Kenya, 2011-2012

\begin{tabular}{|c|c|c|c|c|c|c|}
\hline & \multirow[b]{2}{*}{ Total } & \multirow{2}{*}{$\begin{array}{l}\text { MSM with } \\
\text { any rectal } \\
\text { infection }\end{array}$} & \multicolumn{2}{|c|}{$\begin{array}{l}\text { MSM with } \\
\text { Chlamydia } \\
\text { trachomatis }\end{array}$} & \multicolumn{2}{|c|}{$\begin{array}{l}\text { MSM with } \\
\text { Neisseria } \\
\text { gonorrhoea }\end{array}$} \\
\hline & & & Rectum & Urethra & Rectum & Urethra \\
\hline Symptomatic & 4 & $3(75)$ & - & $1(25)$ & $3(75)$ & $2(50)$ \\
\hline Asymptomatic & 240 & $28(12)$ & $20(8)$ & $14(6)$ & $11(5)$ & $2(1)$ \\
\hline No RAl & 93 & $1(1)$ & $1(1)$ & $4(4)$ & - & - \\
\hline Any RAI & 147 & 27 (18) & $19(14)$ & $10(7)$ & 11 (8) & $2(1)$ \\
\hline
\end{tabular}

documented by the clinician, but was not admitted to in the structured risk assessment.

Sociodemographic and behaviour characteristics of 147 MSM who reported any RAI, with and without rectal infections, are shown in table 2 . The median age of men reporting RAI was 26 years (IQR 23-31), approximately half $(47.6 \%)$ had primary or no education, the majority $(89.1 \%)$ were single, three out of four men $(75.5 \%)$ reported having received money or goods for sex in the past 3 months, about half $(53.1 \%)$ of the men reported sex with men exclusively, and 59 (40.1\%) were HIV-1-infected. Most men (73.5\%) had spent more than 6 months in cohort follow-up. Age, being single, reporting multiple sex partners in the past month and reporting unprotected RAI in the past 6 months were associated with rectal infections in bivariable analysis (at $\mathrm{p}=<0.2$ ). Notably, there were no differences in the prevalence of rectal infection between HIV-1-negative and HIV-1-positive MSM in this analysis (19/88 vs $8 / 59, p=0.2)$. No factor predicted rectal infections in multivariable poisson regression (data not shown).

\section{WHO risk criteria at baseline}

A total of $123(83.7 \%)$ of 147 men reporting any RAI also reported multiple sex partners in the past month; $10(6.8 \%)$ reported a partner with an STI; and 96 (65.3\%) reported unprotected RAI. A total of 85 (57.8\%) MSM reporting any RAI qualified for PT because they both reported unprotected RAI and met one or both of the other two criteria (ie, 79 of the men who reported unprotected RAI also reported multiple partners, four also reported both multiple partners and a partner with an STI, and two also reported a partner with an STI; figure 1A). Eleven MSM met none of the WHO risk criteria for PT (figure 1A).

\section{Predictive values of WHO PT algorithm for asymptomatic rectal infections}

Of all $(n=240)$ the asymptomatic MSM, 85 (35.4\%) met WHO criteria for PT, and 20 (23.5\%) of these $85 \mathrm{MSM}$ had a rectal infection diagnosed. Of 62 men reporting RAI who did not meet WHO criteria, seven $(11.3 \%)$ had a rectal infection diagnosed. The sensitivity and specificity of the WHO algorithm for predicting rectal infection were $74.1 \%$ and $45.8 \%$, respectively. The positive and negative predictive values of the algorithm were $23.5 \%$ and $88.7 \%$, respectively, and the AUC was 0.60 (table 3 ). The predictive values of alternative risk criteria for identifying patients with asymptomatic rectal infections are shown in table 3 . AUCs for these alternatives were similar to those for the WHO algorithm.

\section{Incident rectal infections}

Of 147 asymptomatic MSM reporting RAI at the initial visit, 128 were rescreened after a median 103 days (IQR 93-127), and $16(12.5 \%)$ had an asymptomatic rectal NG or CT infection. Of the 16 incident infections (five NG, nine CT and two NG-CT co-infections), eight (50\%) occurred in men who were treated at baseline. Eleven (69\%) of the 16 infected patients met WHO criteria for PT at 3 months. Reasons for not rescreening 19 of the subjects who reported RAI at baseline included reported migration out of the study area and withdrawal from the study ( $n=7$ and $n=3$, respectively), loss to follow-up $(n=6)$, and missed opportunities for specimen collection $(n=3)$. The incidence was 17.4 (95\% CI 8.3 to 36.4 ) per 100 PY for rectal NG infection, 27.3 (95\% CI 15.1 to 49.3 ) per $100 \mathrm{PY}$ for rectal CT infection, and 39.7 (95\% CI 24.3 to 64.8) per 100 PY for any rectal NG or CT infection. None of the characteristics 
Table 2 Characteristics of 147 MSM who reported receptive anal intercourse and factors associated with prevalent rectal N. gonorrhoeae (NG) or C. trachomatis (CT) infections, coastal Kenya, 2011-2012

\begin{tabular}{|c|c|c|c|c|}
\hline Socio-demographic $\&$ behaviour characteristics & $\begin{array}{l}\text { Total } N=147 \\
\mathrm{n}(\%)\end{array}$ & $\begin{array}{l}\text { Rectal NG or CT infection } \\
\mathrm{N}=27\end{array}$ & $\mathrm{PR}^{*}(95 \% \mathrm{Cl})$ & $\mathrm{p}$ Value \\
\hline Age group (years) & & & & 0.23 \\
\hline $18-24$ & $63(42.9)$ & $16(59.3)$ & Referent & \\
\hline $25-34$ & $70(47.6)$ & $9(33.3)$ & $0.51(0.22-1.15)$ & \\
\hline$>34$ & $14(9.5)$ & $2(7.4)$ & $0.56(0.13-2.458)$ & \\
\hline Education & & & & 0.92 \\
\hline None/Primary & $70(47.6)$ & $13(48.2)$ & Referent & \\
\hline Secondary & $57(38.8)$ & $11(40.7)$ & $1.03(0.47-2.32)$ & \\
\hline Higher/Tertiary & $20(13.6)$ & $3(11.1)$ & $0.81(0.23-2.83)$ & \\
\hline Marital Status & & & & 0.09 \\
\hline Single & $131(89.1)$ & $27(100.0)$ & Referent & \\
\hline Ever married & $16(10.9)$ & $0(0)$ & 4.7 (0.83-infinity) & \\
\hline Received payment for sex & & & & 0.22 \\
\hline No & $36(24.5)$ & $4(14.8)$ & Referent & \\
\hline Yes & $111(75.5)$ & $23(85.2)$ & $1.86(0.64-5.4)$ & \\
\hline Sexual orientation & & & & 0.3 \\
\hline Men only & $78(53.1)$ & $17(63.0)$ & Referent & \\
\hline Men and women & $69(46.9)$ & $10(37.0)$ & $0.66(0.30-1.45)$ & \\
\hline \multicolumn{5}{|l|}{ HIV Status } \\
\hline Negative & $88(59.9)$ & $19(70.4)$ & Referent & 0.26 \\
\hline Positive & $59(40.1)$ & $8(29.6)$ & $0.63(0.27-1.43)$ & \\
\hline Duration of follow-up in cohort & & & & 0.74 \\
\hline $0-1$ months & $22(15.0)$ & $5(18.5)$ & Referent & \\
\hline $1-6$ months & $17(11.6)$ & $4(14.8)$ & $1.04(0.28-3.86)$ & \\
\hline$>6$ & $108(73.5)$ & $18(66.7)$ & $0.73(0.27-1.98)$ & \\
\hline \multicolumn{5}{|c|}{ Risk factors for WHO screening for Presumptive Treatment } \\
\hline Sexual partners last month & & & & 0.17 \\
\hline $0-1$ & $24(16.3)$ & $2(7.4)$ & Referent & \\
\hline$>1$ & $123(83.7)$ & $25(92.6)$ & $2.44(0.58-10.30)$ & \\
\hline Sex partner with an STI last month & & & & 0.49 \\
\hline No & $137(93.2)$ & $26(96.3)$ & Referent & \\
\hline Yes & $10(6.8)$ & $1(3.7)$ & $0.53(0.07-3.88)$ & \\
\hline Unprotected RAI over the past 6 months & & & & 0.06 \\
\hline Yes & $96(65.3)$ & $22(81.5)$ & $2.34(0.89-6.17)$ & \\
\hline No & $51(34.7)$ & $5(18.5)$ & Referent & \\
\hline Met WHO risk criteria for PT $\dagger$ & & & & 0.08 \\
\hline Yes & $85(57.8)$ & $20(74.1)$ & $2.08(0.88-4.93)$ & \\
\hline No & $62(42.2)$ & 7 (25.9) & Referent & \\
\hline
\end{tabular}

presented in table 2 was a significant predictor of incident infection. However, men who had a rectal infection at baseline had a relative hazard of 8.6 (95\% CI 2.9 to 25.4 ) for any incident rectal infection at 3 months. Men who had both a baseline and an incident infection were infected with the same organism in all cases (two NG and six CT infections). Of these eight men, only three (all with CT infections) had requested to be given treatment for their partners.

\section{DISCUSSION}

Over one-third of participating MSM were eligible to receive PT for NG or CT infection according to WHO criteria. The WHO algorithm was 74\% sensitive for detecting rectal infections in 'at-risk' MSM, but had low specificity as expected. For every four MSM meeting the criteria, one infection would be treated in this population. Overall, the WHO algorithm performed poorly, and this would not improve using alternative criteria. While the WHO algorithm currently requires that healthcare workers ask men about three risk factors (ie, unprotected RAI, sex with multiple partners, and partners with an STI), a PT algorithm based only on unprotected RAI in the past 6 months would be easier to use. According to our results, such an algorithm would have a slightly better sensitivity but lower specificity. Similarly to the WHO algorithm, for every four MSM reporting unprotected RAI, one infection would be treated.

Recent studies among mostly MSM sex workers in capital cities of Uganda, Kenya and Cote d'Ivoire reported high (3.1$8.5 \%$ ) rectal NG prevalence, ${ }^{4} 1011$ but data were not presented specifically for MSM reporting RAI. In a recent study by our 

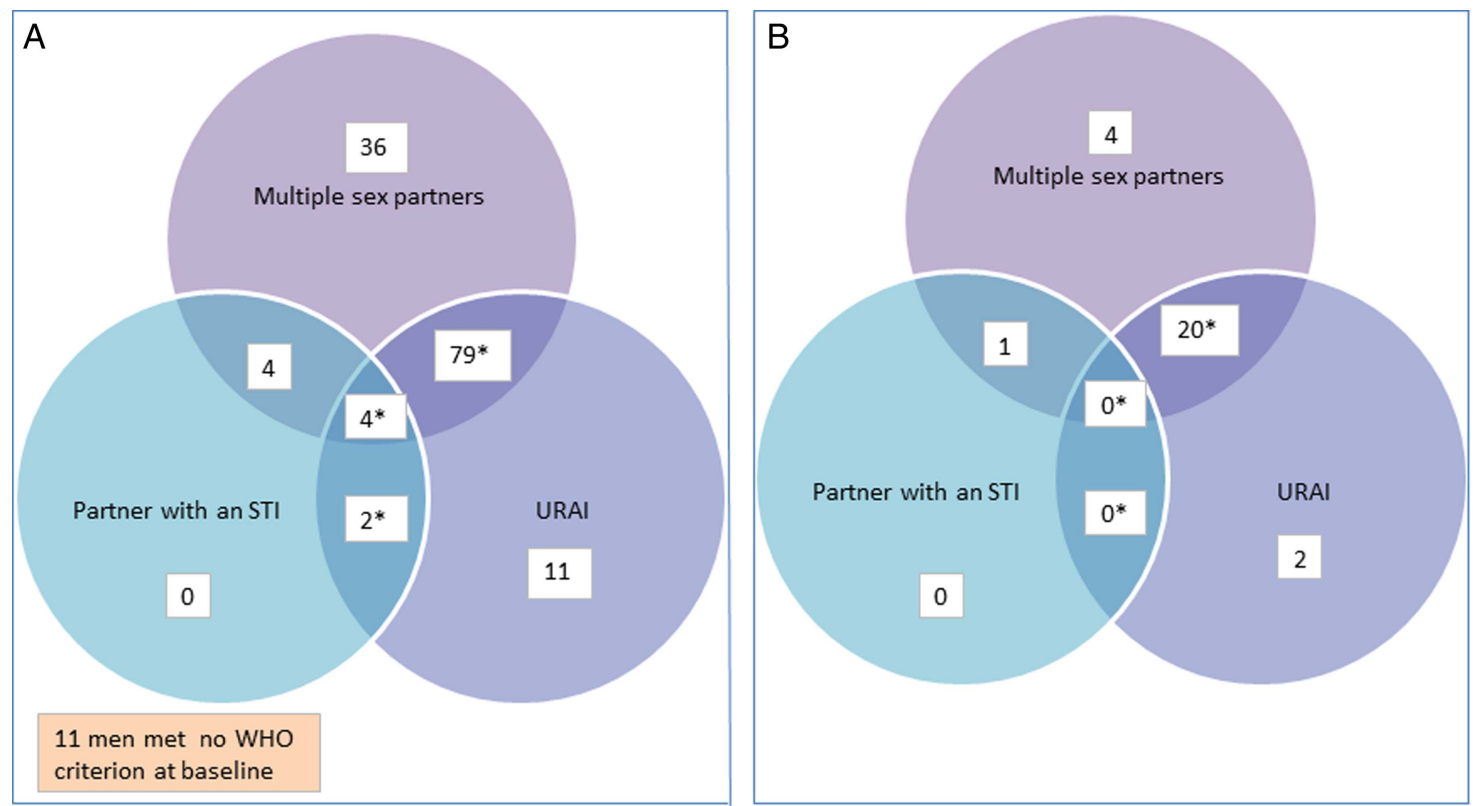

Figure 1 WHO criteria for presumptive treatment of Neisseria gonorrhoeae and Chlamydia trachomatis include reporting (1) unprotected receptive anal intercourse (URAI) and (2) either multiple partners or a partner with a sexually transmitted infection (STI). (A) Distribution of WHO risk criteria (ie, URAI, multiple partners and partner with an STI) among 147 men who have sex with men (MSM) reporting any RAI, Coastal Kenya, $2011-2012$. (B) Distribution of WHO risk criteria (ie, URAl, multiple partners and partner with an STI) among 27 MSM with asymptomatic rectal infections, Coastal Kenya, 2011-2012. Note that none of the 11 men who met no risk criteria had an asymptomatic rectal infection.

Table 3 Predictive values of WHO's presumptive treatment algorithm and alternative criteria for treatment of asymptomatic rectal infections in MSM, Coastal Kenya, 2011-2012

\begin{tabular}{|c|c|c|c|c|c|c|c|}
\hline $\begin{array}{l}\text { MSM }(\mathrm{N}=147) \text { reporting } \\
\mathrm{RAI} \text { and meeting risk } \\
\text { criteria in past } 6 \text { months }\end{array}$ & $\begin{array}{l}\text { MSM with any } \\
\text { rectal infection } \\
(\mathrm{N}=27) \\
\mathrm{N}(\%)\end{array}$ & $\begin{array}{l}\text { MSM without } \\
\text { rectal infection } \\
(\mathrm{N}=120) \\
\mathrm{N}(\%)\end{array}$ & $\begin{array}{l}\text { Sensitivity, } \\
\%(95 \% \mathrm{CI})\end{array}$ & $\begin{array}{l}\text { Specificity, } \\
\%(95 \% \mathrm{CI})\end{array}$ & $\begin{array}{l}\text { PPV, } \\
\%(95 \% \mathrm{Cl})\end{array}$ & $\begin{array}{l}\text { NPV, } \\
\%(95 \% \mathrm{Cl})\end{array}$ & AUC \\
\hline WHO risk criteria* $(\mathrm{N}=85)$ & $20(23.5) \dagger$ & $65(76.5)$ & 74.1 (53.7 to 88.9 ) & 45.8 (36.7 to 55.2 ) & 23.5 (15.0 to 34.0$)$ & 88.7 (78.1 to 95.3$)$ & 0.60 \\
\hline $\begin{array}{l}\text { Did not meet WHO risk } \\
\text { criteria }(\mathrm{N}=62)\end{array}$ & 7 (11.3)‡ & $55(88.7)$ & & & & & \\
\hline Multiple partners $(\mathrm{N}=123)$ & 25 & 98 & $92.6(75.7$ to 99.1$)$ & 18.3 (11.9 to 26.4$)$ & 20.3 (13.6 to 28.5$)$ & 91.7 (73.0 to 99.0$)$ & 0.56 \\
\hline No multiple partners $(\mathrm{N}=24)$ & 2 & 22 & & & & & \\
\hline Unprotected RAI (N=96) & 22 & 74 & 81.5 (62.0 to 93.7$)$ & 38.3 (29.6 to 47.7$)$ & $22.9(15.0$ to 32.6$)$ & 90.2 ( 78.6 to 96.7 ) & 0.60 \\
\hline No unprotected RAI $(\mathrm{N}=51)$ & 5 & 46 & & & & & \\
\hline Partner with STI $(\mathrm{N}=10)$ & 1 & 9 & $3.7(0.1$ to 19.0$)$ & 92.5 (86.2 to 96.5$)$ & 10.0 (0.3 to 44.5$)$ & 81.0 ( 73.4 to 87.2 ) & 0.52 \\
\hline No partner with STI (N=137) & 26 & 111 & & & & & \\
\hline $\begin{array}{l}\text { Multiple partners or partner } \\
\text { with STI }(\mathrm{N}=125)\end{array}$ & 25 & 100 & 92.6 (75.7 to 99.1$)$ & 16.7 (10.5 to 24.6$)$ & $20.0(13.4$ to 28.1$)$ & 90.9 ( 70.8 to 98.9 ) & 0.55 \\
\hline $\begin{array}{l}\text { No multiple partners and no } \\
\text { partner with STI }(\mathrm{N}=22)\end{array}$ & 2 & 20 & & & & & \\
\hline $\begin{array}{l}\text { Multiple partners or } \\
\text { unprotected RAI }(\mathrm{N}=136)\end{array}$ & 27 & 109 & $100.0(87.2$ to 100.0$)$ & 9.2 (4.7 to 15.8 ) & 19.9 (13.5 to 27.6$)$ & $100.0(71.5$ to 100.0$)$ & 0.50 \\
\hline $\begin{array}{l}\text { No multiple partners and no } \\
\text { unprotected } \mathrm{RAI}(\mathrm{N}=11)\end{array}$ & 0 & 11 & & & & & \\
\hline $\begin{array}{l}\text { Unprotected RAI or partner } \\
\text { with STI }(N=100)\end{array}$ & 23 & 77 & 85.2 (66.3 to 95.8$)$ & $35.8(27.3$ to 45.1$)$ & $23.0(15.2$ to 32.3$)$ & 91.5 ( 79.6 to 97.6 ) & 0.61 \\
\hline $\begin{array}{l}\text { No unprotected } \mathrm{RAI} \text { and no } \\
\text { partner with STI }(\mathrm{N}=47)\end{array}$ & 4 & 43 & & & & & \\
\hline
\end{tabular}


group, RAI and symptomatic NG infection in the past 6 months were the strongest predictors of HIV-1 acquisition in MSM, who had an overall HIV-1 incidence of 8.6 (95\% CI 6.7 to 11.0) per 100 PY. As Kenyan MSM are often unaware of the risks that RAI poses for HIV-1 and STI acquisition, ${ }^{12}$ risk-reduction counselling for these men should focus on sexual role-taking and condom use. However, frontline health workers in Kenya lack sensitivity training on male same-sex behaviour and the prevention needs of MSM, and may find it difficult to establish who qualifies for PT or provide effective counselling messages. ${ }^{1} 13$ They also face challenges in offering effective treatment, as national Kenyan guidelines recommend quinolones as a first-line regimen, and wide-spread resistance of NG to quinolones has emerged in Kenya. ${ }^{14}$ Directly observed treatment with ceftriaxone ( $250 \mathrm{mg}$ intramuscularly immediately) or cefixime (400 mg by mouth immediately) and doxycycline (100 mg by mouth twice a week for 7 days) or azithromycin ( $1.0 \mathrm{~g}$ by mouth immediately) are currently the optimal PTs for 'at-risk' MSM meeting WHO criteria.

Fifty per cent of the rectal infections $(n=8)$ we identified at 3 months were possible re-infections or treatment failures. These included two patients with an NG infection who had received directly observed treatment (but no partner treatment), and six men with a CT infection, of whom only three requested treatment for their partners. While some baseline CT infections may have been insufficiently treated because of noncompletion with a 7-day course of doxycycline, it is clear that a more aggressive approach to partner treatment is required. We documented very high 3-month incidences of NG or CT infections in asymptomatic MSM reporting RAI. However, this study was too short to determine the optimal frequency of PT and was not powered to evaluate the effectiveness of PT in reducing the burden of asymptomatic STI among at-risk MSM. Offering PT to MSM reporting RAI without strong emphasis on treating recent sex partners is likely to reduce the effectiveness of a PT programme. A future evaluation of WHO's PT algorithm may be needed to determine the impact of PT plus standard partner referral for STI treatment versus PT with a more intensive approach for partner notification and treatment. ${ }^{15}$ In addition, ongoing surveillance is needed to monitor for drug resistance and ensure that recommended treatment regimens are efficacious.

This study has several limitations. MSM in our study often reported sex work and therefore do not represent MSM outside of the sex trade who may qualify for PT. While these men had access to prevention services through ongoing participation in a research cohort, they may have over-reported protected RAI at repeat visits out of a desire to continue in the 'high-risk' cohort. MSM in our study may also have been more open to report risk behaviour than can be expected at routine care services in Kenya.

In summary, we documented a high burden of rectal NG and CT infections in MSM reporting RAI who had access to STI screening services in Coastal Kenya. While the majority of MSM included in our study had regular risk-reduction counselling, the proportion of men reporting $100 \%$ condom use for all RAI episodes was low, and merits further study. These results support the value of the WHO screening algorithm and recommended PT strategy in this population. However, information on optimal frequency of PT and the overall effectiveness of a PT programme remain elusive. We recommend further evaluation of the impact of PT on the STI burden among MSM and their sexual partners, ideally in conjunction with a partner STI treatment programme.

\section{Key messages}

- The WHO screening algorithm for identification of at-risk men who have sex with men (MSM) for presumptive treatment had $\sim 74 \%$ sensitivity but low specificity for detection of asymptomatic rectal Neisseria gonorrhoeae (NG) and Chlamydia trachomatis (CT) infections.

- In this population of Kenyan MSM, only four who met WHO criteria for presumptive treatment would need to take medication to treat one asymptomatic rectal NG or CT infection.

- A strong emphasis on partner treatment is required for MSM reporting receptive anal intercourse, as $50 \%$ of the incident rectal infections at 3 months were possible re-infections.

\section{Handling editor Jackie A Cassell}

Correction notice The license of this article has also changed since publication to CC BY 4.0.

Acknowledgements We thank staff of the KEMRI-Wellcome Trust Research Programme (KEMRI-WTRP) HIV Key Populations Studies and the International AIDS Vaccine Initiative for supporting the MSM cohort studies. We also thank two anonymous Sexually Transmitted Infections reviewers for useful comments on an earlier version of this paper. The KWTRP at the Centre for Geographical Medicine Research-Kilifi is supported by core funding from the Wellcome Trust (No 077092). This study was made possible by the generous support of the American people through the USA Agency for International Development (USAID). The contents are the responsibility of the International AIDS Vaccine Initiative and do not necessarily reflect the views of USAID or the USA Government. This study is also supported by the University of Washington Center for AIDS Research (CFAR), an NIH funded program (P30 AI027757), which is supported by the following NIH Institutes and Centers (NIAID, NCI, NIMH, NIDA, NICHD, NHLBI, NCCAM). This report was published with permission from KEMRI.

Contributors EJS was primary investigator of this study and wrote the first draft. All authors contributed to the final version of the paper.

Funding International AIDS Vaccine Initiative; NIH, Wellcome Trust. Competing interests None.

Ethics approval The National Ethics Review Committee of the Kenya Medical Research Institute approved this study.

Provenance and peer review Not commissioned; externally peer reviewed.

Open Access This is an Open Access article distributed in accordance with the terms of the Creative Commons Attribution (CC BY 4.0) license, which permits others to distribute, remix, adapt and build upon this work, for commercial use, provided the original work is properly cited. See: http://creativecommons.org/ licenses/by/4.0/

\section{REFERENCES}

1 Beyrer C, Sullivan PS, Sanchez J, et al. A call to action for comprehensive HIV services for men who have sex with men. Lancet 2012;380:424-38.

2 WHO. Prevention and treatment of HIV and other Sexually Transmitted Infections among Men who have Sex with Men and Transgender People. Switzerland: World Health Organization, 2011:1-86.

3 WHO. Sexually Transmitted Infections (STI). 2013. http://appswhoint/iris/bitstream/ 10665/82207/1/WHO_RHR_1302_engpdf (accessed 21 May 2013).

4 Vuylsteke B, Semde G, Sika L, et al. High prevalence of HIV and sexually transmitted infections among male sex workers in Abidjan, Cote d'Ivoire: need for services tailored to their needs. Sex Transm Infect 2012;88:288-93.

5 Sanders EJ, Okuku HS, Smith AD, et al. High HIV-1 incidence, correlates of HIV-1 acquisition, and high viral loads following seroconversion among MSM. AIDS 2013:27:437-46.

6 Okuku HS, Sanders EJ, Nyiro J, et al. Factors associated with herpes simplex virus type 2 incidence in a cohort of human immunodeficiency virus type 1-seronegative Kenyan men and women reporting high-risk sexual behavior. Sex Transm Dis 2011;38:837-44.

7 Sanders EJ, Thiong'o AN, Okuku HS, et al. High prevalence of Chlamydia trachomatis and Neisseria gonorrhoeae infections among HIV-1 negative men who have sex with men in coastal Kenya. Sex Transm Infect 2010;86:440-1.

8 Graham SM, Mugo P, Gichuru E, et al. Adherence to antiretroviral therapy and clinical outcomes among young adults reporting high-risk sexual behavior, 


\section{Clinical}

including men who have sex with men, in coastal kenya. AIDS Behav 2013;17:1255-65.

9 Moncada J, Schachter J, Liska S, et al. Evaluation of self-collected glans and rectal swabs from men who have sex with men for detection of Chlamydia trachomatis and Neisseria gonorrhoeae by use of nucleic acid amplification tests. J Clin Microbiol 2009;47:1657-62.

10 Hladik W, Barker J, Ssenkusu JM, et al. HIV infection among men who have sex with men in Kampala, Uganda_-a respondent driven sampling survey. PLoS One 2012;7:e38143.

11 Muraguri N, Tun W, Okal J, et al. Burden of HIV and sexual behaviour among men who have sex with men and male sex workers in Nairobi, Kenya. Abstract no. MOPE301. XIX International AIDS Conference, Washington DC, 23 July 2012.
12 Geibel S, Luchters S, King'Ola N, et al. Factors associated with self-reported unprotected anal sex among male sex workers in Mombasa, Kenya. Sex Transm Dis 2008;35:746-52.

13 Sullivan PS, Carballo-Dieguez A, Coates T, et al. Successes and challenges of HIV prevention in men who have sex with men. Lancet 2012;380:388-99.

14 Lagace-Wiens PR, Duncan S, Kimani J, et al. Emergence of fluoroquinolone resistance in Neisseria gonorrhoeae isolates from four clinics in three regions of Kenya. Sex Transm Dis 2012;39:332-4.

15 Golden MR, Whittington WL, Handsfield HH, et al. Effect of expedited treatment of sex partners on recurrent or persistent gonorrhea or chlamydial infection. $N$ Engl J Med 2005:352:676-85. 